\title{
Anderson Tower of States and Nematic Order of Spin-1 Bosonic Atoms on a 2D Lattice
}

\author{
Laurent de Forges de Parny, ${ }^{1,2}$ Hongyu Yang, ${ }^{1, *}$ and Frédéric Mila ${ }^{1}$ \\ ${ }^{1}$ Institut de Théorie des Phénomènes Physiques, École Polytechnique Fédérale de Lausanne (EPFL), \\ CH-1015 Lausanne, Switzerland \\ ${ }^{2}$ Laboratoire de Physique, École Normale Supérieure de Lyon, 46 Allée d'Italie, 69364 Lyon Cedex 07, France \\ (Received 10 March 2014; revised manuscript received 20 May 2014; published 12 November 2014)
}

\begin{abstract}
We investigate the structure of the spectrum of antiferromagnetically coupled spin-1 bosons on a square lattice using degenerate perturbation theory and exact diagonalizations of finite clusters. We show that the superfluid phase develops an Anderson tower of states typical of nematic long-range order with broken SU(2) symmetry. We further show that this order persists into the Mott-insulating phase down to zero hopping for one boson per site and down to a critical hopping for two bosons per site, in agreement with mean-field and quantum Monte Carlo results. The connection with the transition between a fragmented condensate and a polar one in a single trap is briefly discussed.
\end{abstract}

Introduction.-Spinor Bose gases have been the subject of very intensive activity over the past 15 years, both experimentally and theoretically [1-4]. For spin-1 bosons, the spin-spin interaction can be ferromagnetic or antiferromagnetic depending on the relative scattering lengths in the $S=0$ and $S=2$ channels, leading in a harmonic trap to a ferromagnetic or a singlet condensate $[5,6]$. When an optical lattice is introduced, the system can, in addition, turn into a Mott insulator at commensurate filling if the tunneling amplitude is small enough as compared to the onsite repulsion. In the single-band approximation at each site, such systems can be described by the Bose-Hubbard Hamiltonian [7,8]

$$
\begin{aligned}
\mathcal{H}= & -t \sum_{\langle i, j\rangle, \sigma}\left(a_{i, \sigma}^{\dagger} a_{j, \sigma}+\text { H.c. }\right)+\frac{U_{0}}{2} \sum_{i} n_{i}\left(n_{i}-1\right) \\
& +\frac{U_{2}}{2} \sum_{i}\left(\vec{S}_{i}^{2}-2 n_{i}\right),
\end{aligned}
$$

where $\langle i, j\rangle$ stands for pairs of nearest neighbors, $\sigma=-1$, 0,1 is the spin, $a_{i, \sigma}^{\dagger}$ and $a_{i, \sigma}$ are creation and annihilation operators of spin- 1 bosons at site $i$, while $n_{i}=\sum_{\sigma} n_{\sigma i}=$ $\sum_{\sigma} a_{\sigma i}^{\dagger} a_{\sigma i}$ and $\vec{S}_{i}$ are the density and spin operators at site $i$. The parameters of this model are the tunneling amplitude $t>0$, the on-site repulsion $U_{0}>0$, and the on-site spinspin interaction $U_{2}$, which is positive (negative) for antiferromagnetic (ferromagnetic) interactions.

The mean-field phase diagram of the antiferromagnetic version of the model has been mapped out quite some time ago by A. Imambekov et al. [8], who found that the odddensity Mott-insulating phases are completely nematic while the even-density ones undergo a transition from a nonmagnetic singlet phase to a nematic phase upon increasing the ratio $t / U_{0}$. In view of the competing orders (such as valence-bond solid order reported in 1D [9-11]), this result clearly calls for further investigations beyond mean field. The first attempt was recently done using the quantum Monte Carlo (QMC) method, which has no minus sign problem for this type of bosonic Hamiltonian [12]. This investigation revealed the presence of a local quadrupolar moment in the entire Mott-insulating phase with one boson per site, while a local quadrupolar moment only develops for large enough hopping in the Mott-insulating phase with two bosons per site. This is consistent with the mean-field phase diagram, but one should keep in mind that the numerical demonstration of nematic long-range order would require an investigation of quadrupolar correlations, which was beyond the scope of Ref. [12]. So further work is definitely needed to check the presence of nematic longrange order in the phase diagram of the model of Eq. (1).

In this Letter, we show that the superfluid phase of spin-1 bosons with antiferromagnetic interactions indeed develops true nematic long-range order in the presence of a lattice. This conclusion is based on a careful investigation of the excitation spectrum of the model using degenerate perturbation theory in the limit $U_{0}=0, U_{2} / t \rightarrow 0$ and exact diagonalizations of finite clusters away from that limit. The key observation is that, in the presence of a lattice, the spectrum acquires the structure of an Anderson tower of states, i.e., a family of low-lying states whose energy collapses onto that of the ground state in the thermodynamic limit, and that all these states have even values of the total spin so that polar states (and not antiferromagnetic states) can be reconstructed as linear combinations of degenerate ground states. Exact diagonalizations are further used to show that this structure persists in the Mottinsulating phase as long as nematic order is present, leading to an alternative determination of the singlet-nematic transition in the $S=2$ Mott insulator.

Let us start by solving the problem analytically in the limit $U_{0}=0, U_{2} / t \rightarrow 0$ which, as we shall show later, turns out to be representative of the general case. Let us 
denote by $N_{s}$ the number of sites and by $N$ the number of bosons. In the noninteracting case $\left(U_{0}=U_{2}=0\right)$, the bosons condense in the $\vec{k}=\overrightarrow{0}$ state, but since there is no magnetic interaction, the spin is irrelevant, and the ground state is vastly degenerate. The ground states are given by

$$
\left|\psi_{n_{-1}, n_{0}, n_{1}}\right\rangle=\prod_{\sigma} \frac{a_{\vec{k}=0, \sigma}^{\dagger n_{\sigma}}}{\sqrt{n_{\sigma} !}}|0\rangle
$$

with $\sum_{\sigma} n_{\sigma}=N$ and $a_{\vec{k}=0, \sigma}^{\dagger}=\left(1 / \sqrt{N_{s}}\right) \sum_{i=1}^{N_{s}} a_{i, \sigma}^{\dagger}$. The degeneracy is equal to $(N+1)(N+2) / 2$.

Let us now consider the effect of $U_{2}$. If $U_{2} / t$ is small, we can use degenerate perturbation theory, which means that we must diagonalize $\sum_{i} \vec{S}_{i}^{2}$ in the subspace spanned by the degenerate ground states of Eq. (2). Now, this operator commutes with the square of the total spin $\vec{S}_{\text {tot }}=\sum_{i} \vec{S}_{i}$. So, in the basis of the eigenstates of $\vec{S}_{\text {tot }}^{2}$, the matrix of $\sum_{i} \vec{S}_{i}^{2}$ is diagonal, and the problem reduces to the evaluation of the expectation value of $\sum_{i} \vec{S}_{i}^{2}$ in the eigenstates of $\vec{S}_{\text {tot }}^{2}$. Since $\sum_{i} \vec{S}_{i}^{2}$ also commutes with the components of $\vec{S}_{\text {tot }}$, hence, with $S_{\text {tot }}^{-}$and $S_{\text {tot }}^{+}$, the expectation value in a state $\left|S_{\text {tot }}, m\right\rangle$ does not depend on $m$, and it is sufficient to calculate it in one member of the family, for instance, $\left|S_{\text {tot }}, m=S_{\text {tot }}\right\rangle$. The calculation of the expectation value of $\vec{S}_{i}^{2}$ in this state can be done analytically (see the Supplemental Material [13]), leading to

$$
\left\langle\vec{S}_{i}^{2}\right\rangle_{S_{\mathrm{tot}}}=\frac{2 N\left(N_{s}-1\right)}{N_{s}^{2}}+\frac{1}{N_{s}^{2}} S_{\mathrm{tot}}\left(S_{\mathrm{tot}}+1\right) .
$$

As anticipated, $\left\langle\vec{S}_{i}^{2}\right\rangle$ is only a function of $S_{\text {tot }}$. This dependence turns out to take the very simple form $S_{\text {tot }}\left(S_{\text {tot }}+1\right)$, but this is by no means a trivial result in the sense that $\sum_{i} \vec{S}_{i}^{2}$ is not simply related to $\vec{S}_{\text {tot }}^{2}$. In fact, $\vec{S}_{\text {tot }}^{2}=\sum_{i} \vec{S}_{i}^{2}+\sum_{i \neq j} \vec{S}_{i} \cdot \vec{S}_{j}$, and the expectation value of $\vec{S}_{i} \cdot \vec{S}_{j}$ in $\left|S_{\text {tot }}, m=S_{\text {tot }}\right\rangle$ does not vanish but is given by

$$
\left\langle\vec{S}_{i} \cdot \vec{S}_{j}\right\rangle_{S_{\mathrm{tot}}}=-\frac{2 N}{N_{s}^{2}}+\frac{1}{N_{s}^{2}} S_{\mathrm{tot}}\left(S_{\mathrm{tot}}+1\right) .
$$

Equation (3) implies in particular that, in the total singlet, and in the thermodynamic limit $N, N_{s} \rightarrow+\infty, \rho=N / N_{s}$ fixed, the local value of the square of the spin is given by

$$
\left\langle\vec{S}_{i}^{2}\right\rangle_{S_{\mathrm{tot}}=0}=2 \rho,
$$

where $\rho$ is the boson density. Contrary to what one might naively expect, this limiting value is not of the form $S(S+1)$ for some integer $S$. It is, however, in good agreement with the QMC results obtained for one and two bosons per site [12]. Finally, let us emphasize that, in
Eq. (3), $S_{\text {tot }}$ can only take even values because it corresponds to the total spin of spin-1 bosons in a single mode, the $\vec{k}=\overrightarrow{0}$ one.

Coming back to the Hamiltonian of Eq. (1) in the limit $U_{0}=0, U_{2} / t \rightarrow 0$, the low-energy spectrum is, thus, given by

$$
E_{S_{\mathrm{tot}}}=-4 t N-\frac{N}{N_{s}} U_{2}+\frac{U_{2}}{2} \frac{1}{N_{s}} S_{\mathrm{tot}}\left(S_{\mathrm{tot}}+1\right),
$$

where the first term is the energy of the noninteracting condensate. The important property is that the slope is proportional to $1 / N_{s}$ and tends to zero in the thermodynamic limit, leading to a quasidegenerate ground state. In quantum antiferromagnets, this property goes under the name of Anderson's tower of states [16-20]: on the basis of the low-lying states of this tower, it is possible to reconstruct a wave function very close to the Néel state with spins up on one sublattice and down on the other sublattice whose energy is very low and scales to the ground-state energy when the system size increases so that the appearance of a tower of states in the low-energy spectrum indicates that the $\mathrm{SU}(2)$ symmetry is spontaneously broken in the ground state in favor of antiferromagnetism.

Note that the tower of states remains a well-defined concept as long as the energies of the states building this tower are well separated from those of the elementary excitations, which also tend to the ground-state energy in ordered systems. In the present calculation, which is performed in the $U_{2} / t \rightarrow 0$ limit, this is clearly true since the elementary excitations consist in exciting a particle out of the condensate with an energy of order $t / N_{s}$ for small wave vector, while the states of the tower have energies of order $U_{2} / N_{s}$.

Now, using only states with small values of $S_{\text {tot }}$, it is possible to reconstruct almost exactly polar states. Indeed, all polar states are related by a rotation to $\left|\psi_{0, N, 0}\right\rangle \propto a_{\vec{k}=\overrightarrow{0}, 0}^{\dagger N}|0\rangle$, and this state can be expanded in the basis of eigenstates of $\vec{S}_{\text {tot }}^{2}$ as

$$
\left|\psi_{0, N, 0}\right\rangle=\sum_{S=0,2, \ldots, N} c_{N}(S)|S, m=0\rangle .
$$

The coefficients $c_{N}(S)$ can be determined analytically (Supplemental Material [13]) and are given by

$$
c_{N}(S)=\sqrt{\frac{(2 S+1) N !}{(N-S) ! !(N+S+1) ! !}} .
$$

These coefficients only take significant values up to $S=O(\sqrt{N})$ : as shown in Fig. 1, the maximum value of the spin $S_{N}$ up to which one has to sum to satisfy the sum rule $\sum_{S}\left|c_{N}(S)\right|^{2}=1$ to a given accuracy scales as $\sqrt{N}$. As a consequence, the polar state $\left|\psi_{0, N, 0}\right\rangle$ has an energy per site 


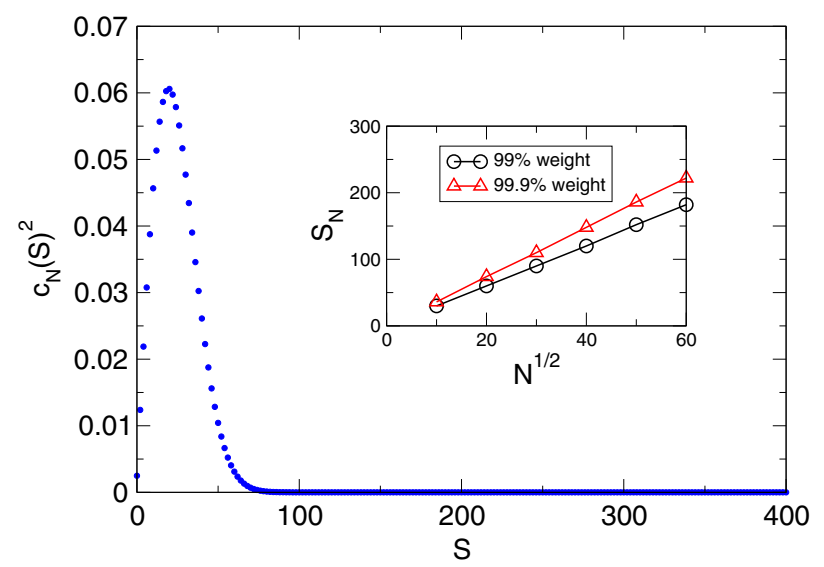

FIG. 1 (color online). Squares $c_{N}(S)^{2}$ of the coefficients of the expansion of the polar state $\left|\psi_{0, N, 0}\right\rangle$ in the eigenstates $|S, m=0\rangle$ of $\vec{S}_{\text {tot }}^{2}$ as a function of $S$ for $N=400$. Inset: value of the spin $S_{N}$ up to which one has to sum to satisfy the sum rule $\sum_{S}\left|c_{N}(S)\right|^{2}=$ 1 to a given accuracy. It scales as $\sqrt{N}$.

that scales to the ground state one as $1 / N_{s}$ in the thermodynamic limit. It is, thus, a ground state in that limit, which proves the presence of long-range nematic order. These results establish that, in the limit $U_{0}=0, U_{2} / t \rightarrow 0$, the $\mathrm{SU}(2)$ symmetry of the superfluid ground state of spin-1 bosons on a lattice is spontaneously broken in favor of nematic order.

It is instructive to compare these results to the case of spin-1 bosons in a trap [21-25]. In the single-mode approximation, the Hamiltonian reads

$$
\mathcal{H}=\frac{U_{s}}{2 N} \vec{S}^{2},
$$

where $U_{s}$ is the spin interaction energy per atom. The ground state is a nondegenerate singlet with energy $E_{0}=0$, and the excitation energies are given by $E_{S}=$ $U_{s} /(2 N) S(S+1), S=2,4, \ldots$. The structure of this spectrum is similar to that of Eq. (6), with in particular a slope that goes to zero as $1 / N$ in the thermodynamic limit. Accordingly, the consequences in that limit are very similar: As discussed in Refs. [21-25], spontaneous symmetry breaking takes place since a polar state can be stabilized for infinitesimal quadratic Zeeman coupling coupling in the thermodynamic limit. The decomposition of the polar state into the angular momentum basis also takes a very similar form, our analytical result of Eq. (7) corresponding to the large- $q$ limit of the result of Ref. [25], where the transition between a fragmented condensate and a polar state induced by a quadratic Zeeman coupling $q$ has been investigated in detail.

Let us now turn to the general case. The phase diagram has been previously studied using mean-field theory [8,26,27], variational Monte Carlo method [28], and quantum Monte Carlo simulations [12,29], and some exact

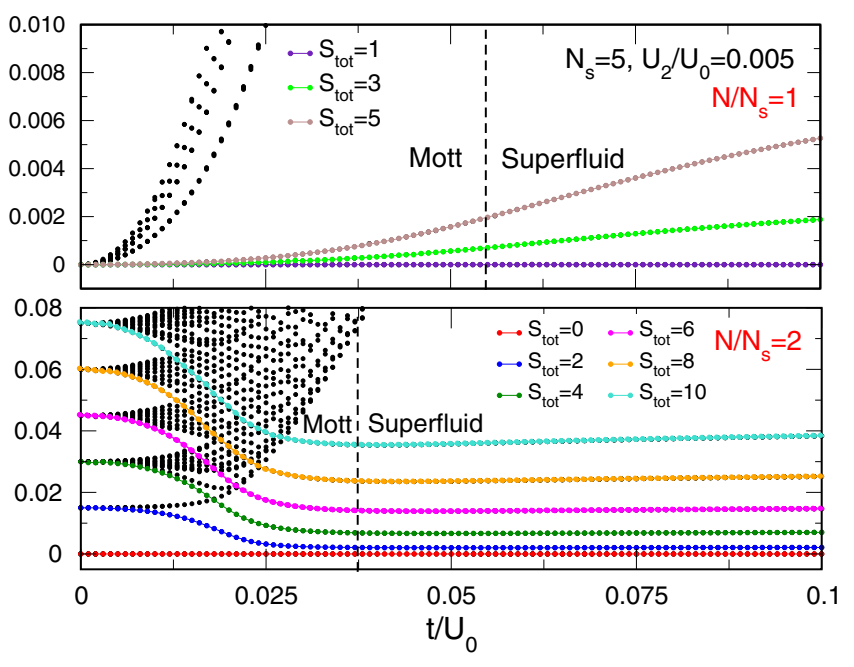

FIG. 2 (color online). Low-energy spectra for five sites with $N / N_{s}=1$ (top) and $N / N_{s}=2$ (bottom) with $U_{2} / U_{0}=0.005$. The energies are in unit of $U_{0}$ and measured from the ground state. The vertical dashed line indicates the Mott-superfluid transition according to the QMC simulations [12].

results have been established [30]. These methods have led to the conclusion that, for integer filling, there is a superfluid-insulator Mott transition upon increasing $U_{0} / t$, and the insulating state is always nematic for odd filling while there is an additional nematic-singlet transition upon further increasing $U_{0} / t$ for even filling. Now that we have analytically demonstrated that the superfluid state is nematic in the limit $U_{0} / t=0$ on the basis of the structure of the low-energy spectrum, it is natural to ask to which extent this structure persists away from that limit. For that purpose, we have performed exact diagonalizations of finite-size clusters for one and two bosons per site, with up to 10 and 8 sites, respectively. The low-energy spectra for five sites are depicted in Fig. 2 as a function of $t / U_{0}$. In both cases, the total spin $S_{\text {tot }}$ changes by 2 from one state to the next, and the energy of a state measured from the ground state is proportional to $S_{\text {tot }}\left(S_{\text {tot }}+1\right)$, with a coefficient that tends to $U_{2} / 2 N_{s}$ in the large- $t / U_{0}$ limit, in agreement with Eq. (6). This structure persists below the superfluid-insulator transition without any hint that the system undergoes a phase transition. This suggests that the nematic order predicted previously in the insulating phases is continuously related to the nematic order we have established in the $U_{0}=0, U_{2} / t \rightarrow 0$ limit. Upon further reducing the ratio $t / U_{0}$, the structure of the tower of states remains essentially unaffected for one boson per site, but a series of level crossings leads to a completely different spectrum for two bosons per site, which signals a nematicsinglet transition. The same structure has been observed on larger clusters (Supplemental Material [13]).

In the Mott-insulating phase, the identification of the order as nematic and not antiferromagnetic actually deserves special attention since, for antiferromagnetic 


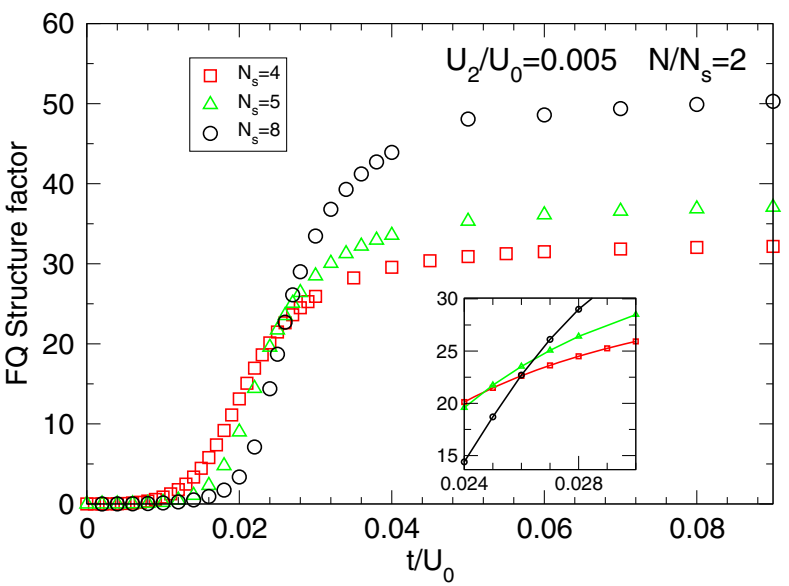

FIG. 3 (color online). Ferromagnetic quadrupolar structure factor for four-, five-, and eight-site clusters with two bosons per site.

coupled spins, one might in general expect simple Néel order on a bipartite lattice. In exact diagonalizations, antiferromagnetic and nematic order can be distinguished by the quantum numbers that appear in the tower of states. For Néel order, all values of $S_{\text {tot }}$ are represented in the tower of states because, to reconstruct the Néel state with up spins on one sublattice and down spins on the other one, one needs states with both even and odd total spin, whereas for quadrupolar order, one only needs states with even total spin [31,32]. In view of the analytical results of the $U_{0}=0, U_{2} / t \rightarrow 0$ limit, we expect by continuity the low-lying states calculated by exact diagonalizations away from that limit to carry only even or odd total spin. We have explicitly checked this to be the case for the five-site cluster. So the fact that only even steps in $S_{\text {tot }}$ appear in the tower of states is an additional confirmation that, in the regions of the Mott-insulating phases with spontaneously broken $\mathrm{SU}(2)$ symmetry, the order is indeed nematic.

As an independent confirmation, we have calculated the ferroquadrupolar structure factor $S^{Q}(\vec{k}=\overrightarrow{0})$, with $S^{Q}(\vec{k})=\sum_{j} \exp \left(i \vec{k} \cdot \vec{r}_{j}\right)\left\langle\vec{Q}_{0} \cdot \vec{Q}_{j}\right\rangle$, where the quadrupolar operator is defined by $\vec{Q}=\left\{\left(S^{x}\right)^{2}-\left(S^{y}\right)^{2}, 1 / \sqrt{3}\left[2\left(S^{z}\right)^{2}-\right.\right.$ $\left.\left.\left(S^{x}\right)^{2}-\left(S^{y}\right)^{2}\right], S^{x} S^{y}+S^{y} S^{x}, S^{y} S^{z}+S^{z} S^{y}, S^{z} S^{x}+S^{x} S^{z}\right\}$. For two bosons per site, as can be seen in Fig. 3, it increases with the size for large enough $t / U_{0}$, and it decreases with the size for small enough $t / U_{0}$. The crossing point can be taken as an approximation of the transition to nematic order (Supplemental Material [13]), and the critical value $t_{c} / U_{0} \sim 0.026$ is in excellent agreement with the QMC estimate based on the development of a local quadrupolar moment.

In principle, it is possible to locate the singlet-nematic transition just by investigating the spin gap, which is expected to be finite in the singlet phase and to scale to zero in the nematic phase. It turns out that this is not very accurate for the sizes accessible with exact

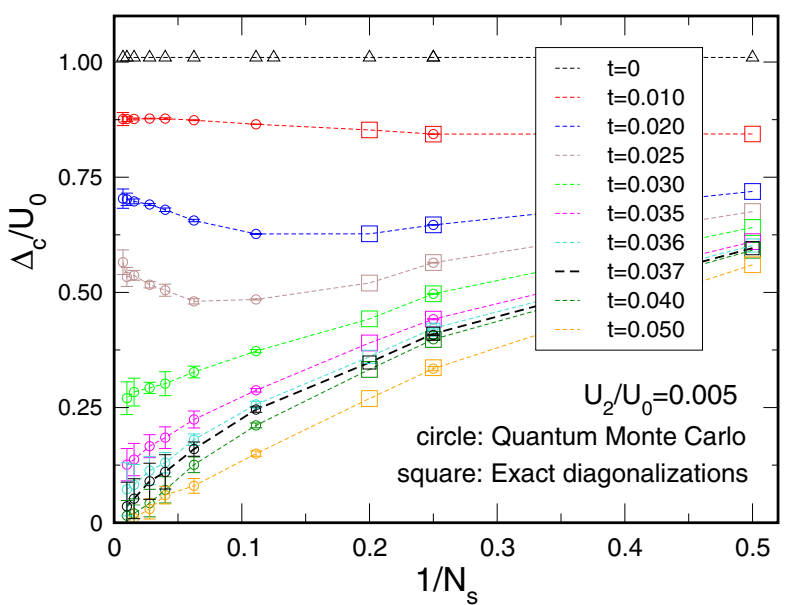

FIG. 4 (color online). Charge gap as a function of the inverse number of sites for various values of $t / U_{0}$. It opens at $t_{c} / U_{0} \simeq 0.037$, which marks the superfluid-insulator transition.

diagonalizations, and only a very rough estimate of the transition can be obtained along these lines (Supplemental Material [13]). This estimation, however, is still consistent with other estimates.

Finally, we have attempted to locate the superfluidinsulator transition, which, as usual, corresponds to the opening of the charge gap defined by $\Delta_{c}=E(N+1)+$ $E(N-1)-2 E(N)$. As for the singlet-triplet gap, the results are consistent with the QMC estimate $\left(t_{c} / U_{0} \simeq 0.037\right)$, but the sizes accessible to exact diagonalizations do not lead to a very precise estimate. It is only with the help of QMC calculations, with the stochastic Green function algorithm [33], of the energies for larger system sizes that this criterion can be shown to coincide with the appearance of a superfluid stiffness [12] (see Fig. 4). Note that, for $U_{2} / U_{0}=0.005$, the superfluid-Mott insulator transition is well separated from the singletnematic transition. Increasing $U_{2}$ pushes the singletnematic transition to larger critical value, and when $U_{2} / U_{0} \sim 0.1$, there is only one transition from the singlet Mott phase to the nematic superfluid left (Supplemental Material [13]).

To summarize, let us put the present results in perspective. Except in one dimension, where the density matrix renormalization group can be used [10,34,35], the investigation of lattice bosonic models is largely dominated by QMC, and rightly so since, due to the absence of minus sign problem in many cases, extremely accurate results can be obtained on very large system sizes. Yet, as demonstrated in the present Letter, investigating the excitation spectrum of the model with analytical tools if possible, or with exact diagonalizations of small clusters, can lead to very interesting insight into the properties of the system, even if the sizes accessible are much smaller than those with QMC. In the present case, the structure of the lowenergy spectrum, which consists of an Anderson tower of 
state in a large portion of the phase diagram, is an extremely fruitful piece of information. In particular, it has led to the demonstration that, in the superfluid phase of spin-1 bosons on a lattice, the $\mathrm{SU}(2)$ symmetry is spontaneously broken, by contrast to the case of bosons in a single mode, which require an $\mathrm{SU}(2)$ symmetry-breaking interaction to build a polar condensate. It will be very interesting to investigate the implications of this result on the dynamics of spinor condensates [36] in the presence of a lattice.

We thank G. Batrouni, F. Hébert, A. Läuchli, and V. G. Rousseau for useful discussions and T. Roscilde for his critical reading of the manuscript. We are especially indebted to F. Gerbier for insightful remarks on the thermodynamic limit in a single trap and to an anonymous referee for a simpler proof of Eq. (3). This work was supported by the Swiss National Science Foundation.

*hongyu.yang@epfl.ch

[1] D. M. Stamper-Kurn and W. Ketterle, Coherent Matter Waves, edited by R. Kaiser, C. Westbrook, and F. David (Springer, Berlin, Heidelberg, 2001), Chap. 2, pp. 139-217.

[2] Y. Kawaguchi and M. Ueda, Phys. Rep. 520, 253 (2012).

[3] M. Lewenstein, A. Sanpera, V. Ahufinger, B. Damski, A. Sen(De), and U. Sen, Adv. Phys. 56, 243 (2007).

[4] D. M. Stamper-Kurn and M. Ueda, Rev. Mod. Phys. 85, 1191 (2013).

[5] T.-L. Ho, Phys. Rev. Lett. 81, 742 (1998).

[6] T. Ohmi and K. Machida, J. Phys. Soc. Jpn. 67, 1822 (1998).

[7] D. Jaksch, C. Bruder, J. I. Cirac, C. W. Gardiner, and P. Zoller, Phys. Rev. Lett. 81, 3108 (1998).

[8] A. Imambekov, M. Lukin,, and E. Demler, Phys. Rev. A 68, 063602 (2003).

[9] F. Zhou and M. Snoek, Ann. Phys. (Amsterdam) 308, 692 (2003).

[10] M. Rizzi, D. Rossini, G. De Chiara, S. Montangero, and R. Fazio, Phys. Rev. Lett. 95, 240404 (2005).

[11] V. Apaja and O.F. Syljuåsen, Phys. Rev. A 74, 035601 (2006).

[12] L. de Forges de Parny, F. Hébert, V. G. Rousseau, and G. G. Batrouni, Phys. Rev. B 88, 104509 (2013).

[13] See the Supplemental Material at http://link.aps.org/ supplemental/10.1103/PhysRevLett.113.200402, which includes Refs. [12,14,15], for the analytical calculation of the tower of states in the limit $U_{0}=0, U_{2} / t \rightarrow 0$, and for additional information on the numerical data (excitation spectrum of larger systems, finite-size analysis of the ferroquadrupolar structure factor, of the level crossings, and of the spin gap).

[14] S. Liang, Comput. Phys. Commun. 92, 11 (1995).

[15] H. Q. Lin, Phys. Rev. B 42, 6561 (1990).

[16] P. W. Anderson, Phys. Rev. 86, 694 (1952).

[17] B. Bernu, C. Lhuillier, and L. Pierre, Phys. Rev. Lett. 69, 2590 (1992).

[18] B. Bernu, P. Lecheminant, C. Lhuillier, and L. Pierre, Phys. Rev. B 50, 10048 (1994).

[19] C. Lhuillier, arXiv:cond-mat/0502464.

[20] G. Misguich and P. Sindzingre, J. Phys. Condens. Matter 19, 145202 (2007).

[21] T.-L. Ho and S. K. Yip, Phys. Rev. Lett. 84, 4031 (2000).

[22] M. Koashi and M. Ueda, Phys. Rev. Lett. 84, 1066 (2000).

[23] E. J. Mueller, T. L. Ho, M. Ueda, and G. Baym, Phys. Rev. A 74, 033612 (2006).

[24] H. Tasaki, Phys. Rev. Lett. 110, 230402 (2013).

[25] L. D. Sarlo, L. Shao, V. Corre, T. Zibold, D. Jacob, J. Dalibard, and F. Gerbier, New J. Phys. 15, 113039 (2013).

[26] T. Kimura, S. Tsuchiya, and S. Kurihara, Phys. Rev. Lett. 94, 110403 (2005).

[27] R. V. Pai, K. Sheshadri, and R. Pandit, Phys. Rev. B 77, 014503 (2008).

[28] Y. Toga, H. Tsuchiura, M. Yamashita, K. Inaba, and H. Yokoyama, J. Phys. Soc. Jpn. 81, 063001 (2012).

[29] G. G. Batrouni, V. G. Rousseau, and R. T. Scalettar, Phys. Rev. Lett. 102, 140402 (2009).

[30] H. Katsura and H. Tasaki, Phys. Rev. Lett. 110, 130405 (2013).

[31] N. Shannon, T. Momoi, and P. Sindzingre, Phys. Rev. Lett. 96, 027213 (2006).

[32] K. Penc and A. Läuchli, in Introduction to Frustrated Magnetism, edited by C. Lacroix, P. Mendels, and F. Mila (Springer, Berlin, Heidelberg, 2011), Chap. 13, pp. 331-362.

[33] V. G. Rousseau, Phys. Rev. E 78, 056707 (2008).

[34] S. Bergkvist, I. P. McCulloch, and A. Rosengren, Phys. Rev. A 74, 053419 (2006).

[35] K. Rodríguez, A. Argüelles, A. K. Kolezhuk, L. Santos, and T. Vekua, Phys. Rev. Lett. 106, 105302 (2011).

[36] A. Lamacraft, Phys. Rev. B 81, 184526 (2010). 